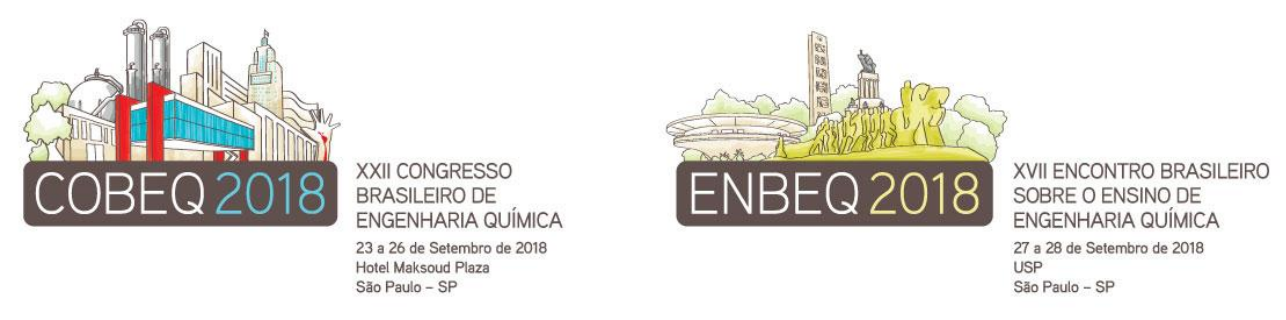

\title{
Produção de adsorvente proveniente de resíduos sólidos agrícolas para remoção de cromo hexavalente em águas residuais
}

\author{
BAIERLE A.L ${ }^{1}$, COLLAZZO G.C ${ }^{1}$ \\ ${ }^{1}$ Universidade Federal de Santa Maria, Centro de Tecnologia, Departamento de Engenharia \\ Química \\ E-mail para contato: anabaierle123@gmail.com
}

\begin{abstract}
RESUMO - A remoção de Cr (VI) de águas residuais apresenta grande importante devido aos danos que esse composto pode causar. Este trabalho tem como objetivo estudar a remoção de $\mathrm{Cr}$ (VI) de águas residuais a partir da produção de um adsorvente proveniente de resíduos sólidos agrícolas. Para isso, estudou-se o biochar de lichia, analisou-se a área superficial, e observou-se que o mesmo apresenta 37,275 $\mathrm{m}^{2} / \mathrm{g}$ de área. Pelo ensaio de adsorção, observou-se que o adsorvente removeu entre 40 e $95 \%$ do composto. Além disso, notou-se que o modelo cinético que melhor representa o processo foi o modelo de pseudo-primeira ordem.
\end{abstract}

\section{INTRODUÇÃO}

Adsorventes de baixo custo provenientes dos setores agrícola vêm sendo reconhecidos como uma alternativa para o tratamento de águas residuais uma vez que a remoção de poluentes de difícil degradação é dispendiosa. Devido a crescente industrialização, tem-se o problema de poluição com metais pesados. Em sistemas aquosos, o $\mathrm{Cr}$ existe tanto na forma $\mathrm{Cr}$ (VI) como na $\mathrm{Cr}$ (III), o Cr (VI) é extremamente tóxico e cancerígeno podendo causar danos mesmo em baixas concentrações (Qi et al., 2016).

O processo de adsorção é considerado uma boa alternativa no tratamento de águas residuais devido à facilidade de operação e simplicidade de projeto. $\mathrm{O}$ adsorvente mais comumente utilizado é o carvão ativado. No entanto, a sua utilização generalizada no tratamento de águas residuais é por vezes restringida devido ao seu custo mais elevado e capacidade de regeneração do adsorvente (Gautam et al., 2014).

Uma grande variedade de adsorventes de baixo custo vem sendo avaliada para a remoção de vários tipos de poluentes das águas residuais (Chao et al., 2017). Por essa razão, as pesquisas sobre o uso de adsorventes de baixo custo, disponíveis localmente, reutilizáveis e de fontes naturais vêm sendo cada vez mais crescente.

Considerando que o Brasil é um importante produtor de frutas e que o processamento de grande parcela dessas frutas gera elevada quantidade de resíduos sólidos, muitas vezes, descartados de forma inadequada, por ter baixo valor econômico, o objetivo do presente trabalho é utilizar os resíduos, na forma de cascas e caroços de Litchi chinensis (Sapindaceae), com vistas à remoção de $\mathrm{Cr}$ (VI) através de processo de adsorção. 


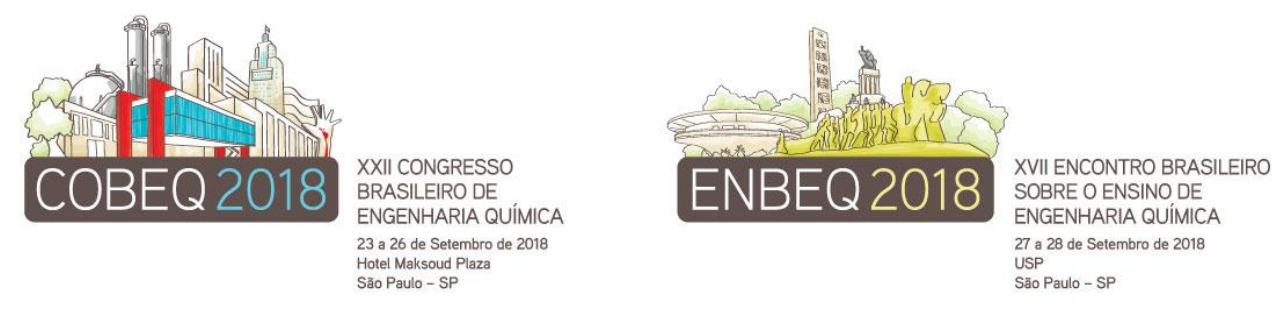

\section{MATERIAIS E MÉTODOS}

\subsection{Obtenção e pré-tratamento}

Para os ensaios de adsorção foi utilizada uma amostra de resíduos sólidos proveniente do processamento da lichia (cascas e caroço), obtida de produtores da região sul do Brasil. A amostra foi lavada com água deionizada e seca em estufa a $60{ }^{\circ} \mathrm{C}$, durante 8 horas. Em seguida a amostra foi moída em moinho de sapatas, peneirada e selecionada em uma fração com diâmetro abaixo de $710 \mu \mathrm{m}$.

\subsection{Produção do biochar}

O biochar foi produzido a partir do processo de pirolise em um forno tubular de marca Sanchis e um reator de quartzo. Na figura 1 pode ser observado um esquema do aparato experimental. $\mathrm{O}$ procedimento consistiu em aquecer a amostra (cascas e caroços de Lichia previamente preparadas até $800{ }^{\circ} \mathrm{C}$ (taxa de $25{ }^{\circ} \mathrm{C} / \mathrm{min}$ e isoterma de $30 \mathrm{~min}$ ) utilizando nitrogênio industrial a $1 \mathrm{~L} / \mathrm{min}$. Após o tempo de isoterma o sistema era então resfriado até a temperatura de $50^{\circ} \mathrm{C}$, e fração sólida removida. Essa fração era então moída e armazenada para posterior utilização. Utilizou-se um analisador de área superficial da Quantachrome Instruments (Nova 1200e, USA) para caracterizar o biochar. A área de superfície foi determinada segundo método de Brunauer, Emmett, e Teller (BET) e a distribuição de tamanho de poros foi obtida pelo método Barret, Joyner e Halenda (BJH).

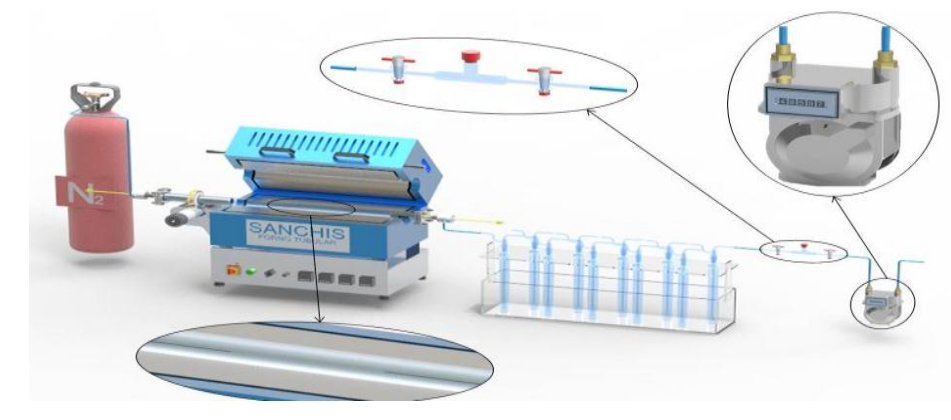

Figura 1 - Aparato experimental composto de forno horizontal, reator tubular de quartzo, cilindro de nitrogênio e sistema de condensação dos gases produzidos na pirólise com medidor de fluxo de gás e coletor na saída.

\subsection{Ensaios de Adsorção}

Os ensaios de adsorção foram conduzidos em diferentes concentrações (20, 40, 60 e 80 $\mathrm{mg} / \mathrm{L}$ ) de uma solução de $\mathrm{K}_{2} \mathrm{Cr}_{2} \mathrm{O}_{7}$. Os ensaios ocorreram utilizando um banho termostático por 120 min e agitação de $250 \mathrm{rpm}$. Em cada solução de $50 \mathrm{~mL}$, com o pH ajustado para 2,5, foi adicionado $0,1 \mathrm{~g}$ de adsorvente. Alíquotas de $10 \mathrm{~mL}$ foram retiradas em tempos prédeterminados e analisadas em espectrofotômetro (Biospectro, SP-22, Brasil) em $540 \mathrm{~nm}$. O Cr (VI) foi quantificado pelo método de complexação com 1,5-difenilcarbazida. Os modelos foram ajustados utilizando analise de regressão não-linear e os parâmetros o $\mathrm{R}^{2}$ e quiquadrado $\left(\mathrm{X}^{2}\right)$ fora utilizados para avaliação dos resultados. 

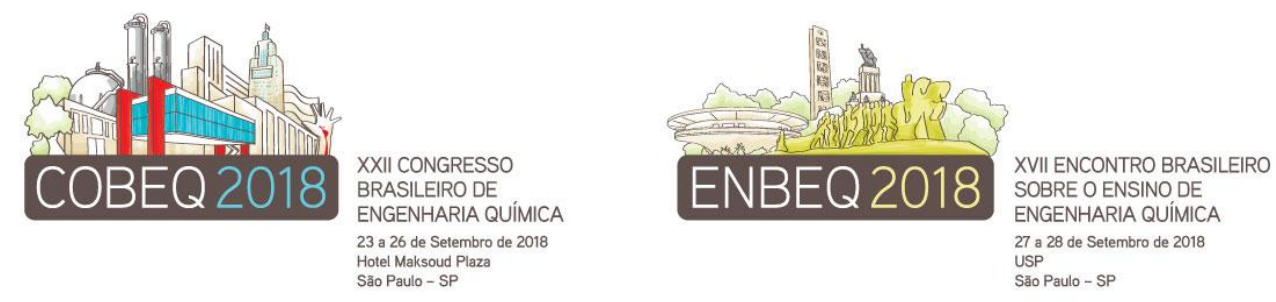

\section{RESULTADOS E DISCUSSÃO}

$\mathrm{O}$ adsorvente (biochar) produzido apresentou uma área de superfície de $37,275 \mathrm{~m}^{2} / \mathrm{g} \mathrm{e}$ volume total de poros de $0,02705 \mathrm{~cm}^{3} / \mathrm{g}$. Dos resultados pode-se afirmar que o biochar consiste em uma estrutura mesoporosa com poros de tamanho médio de $14.5 \AA$.

Dos ensaios de adsorção (Figura 2) observou-se que equilíbrio foi atingido em 120 minutos e os maiores valores de capacidade de remoção foram para as maiores concentrações. Em termos de porcentagem de remoção os valores variaram entre 40 e $95 \%$. Estes resultados mostram que o biochar em estudo tem um grande potencial como adsorvente de $\mathrm{Cr}$ (VI), uma vez que apresenta área superficial relativamente baixa comparando com carvão ativado comercial. Entre os modelos de equilíbrio foram avaliados de acordo com a isoterma de Langmuir (Langmuir, 1918), Freundlich (Freundlich, 1906) e Sips (Zarei, 2018), resultados mostrados na Figura 3 (a). Os dados de equilíbrio são melhores representados por Freundlich $\left(\mathrm{r}^{2}=0,92\right.$ e $\left.\mathrm{X}^{2}=0,52\right)$. Com relação a cinética de adsorção do $\mathrm{Cr}$ (VI) no biochar foram ajustados os modelos de pseudo-primeira ordem (Lagergren, 1898), pseudo-segunda ordem (Ho, 1998) e Elovich (Qiu et al., 2009). Dos ajustes, observou-se que de acordo com o coeficiente de correlação a ordem de ajuste foi pseudo-primeira ordem > Elovich > pseudosegunda ordem. $\mathrm{O}$ ajuste do modelo de pseudo-primeira ordem está mostrado na Figura 3 (b).

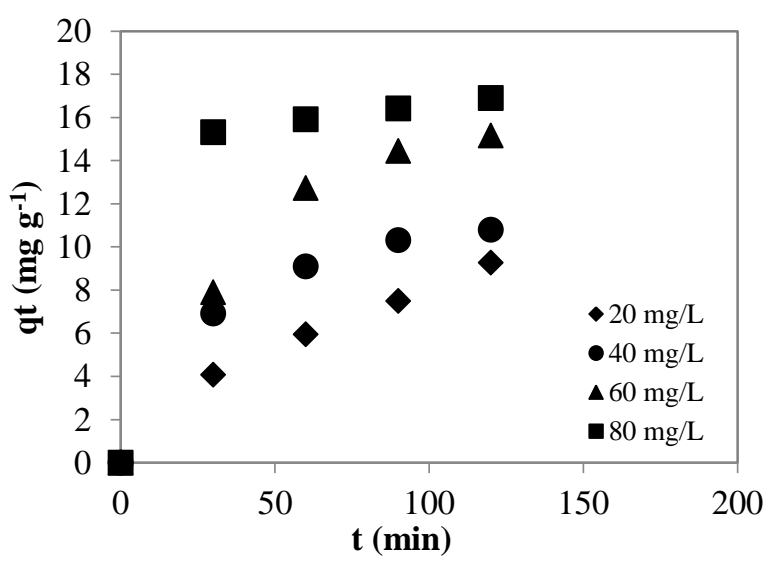

Figura 2 - Capacidade de adsorção em função do tempo.
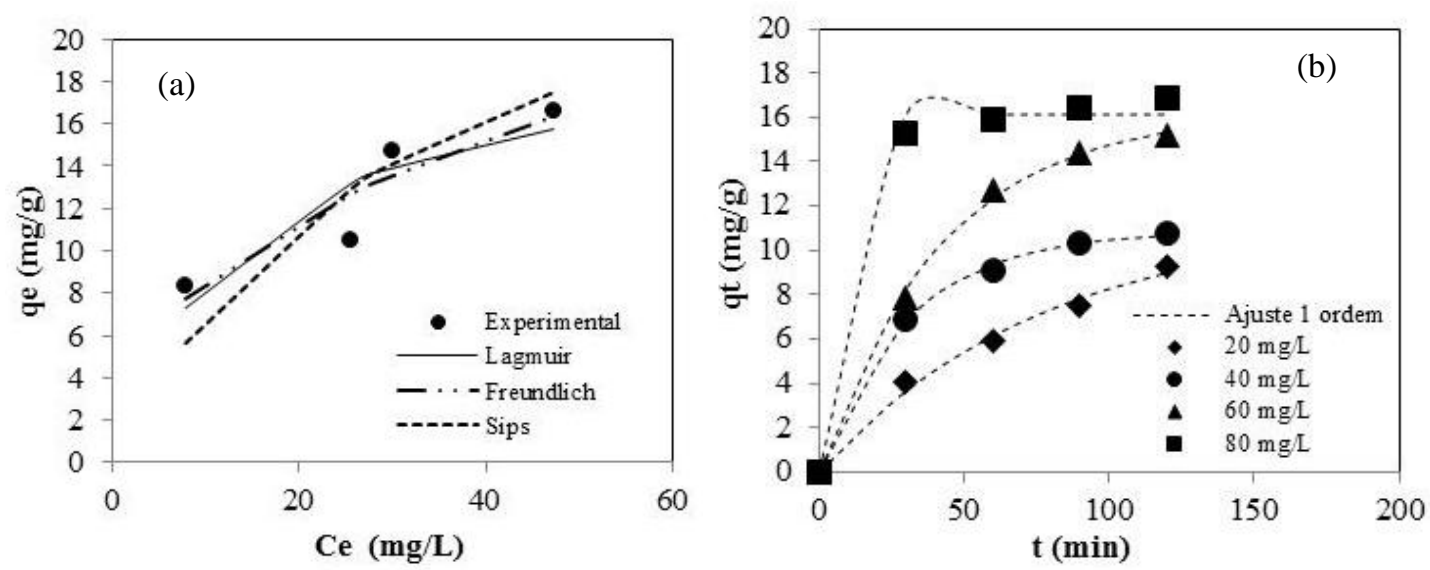


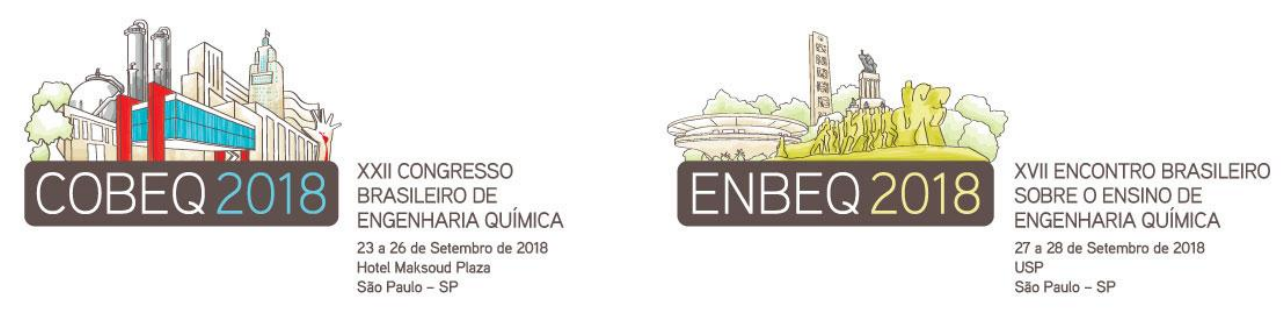

Figura 3 - (a) Ajuste das isotermas de equilíbrio, (b) Ajuste dos modelos cinéticos.

\section{CONCLUSÃO}

A utilização de adsorventes alternativos para a remoção de componentes tóxicos em águas residuais torna-se muito atrativa pelo baixo custo e facilidade de sua aquisição. $\mathrm{O}$ biochar da casca e caroço de lichia obteve área de superfície de $37,275 \mathrm{~m}^{2} / \mathrm{g}$ e volume total de poros de $0,02705 \mathrm{~cm}^{3} / \mathrm{g}$. Pelos ensaios de adsorção, notou-se que o biochar obteve em termos de porcentagem de remoção entre 40 e $95 \%$. Assim, pode-se afirmar que a aplicação desse resíduo diminuirá os impactos ambientais em consequência do seu descarte, promoverá um impacto tecnológico positivo com sua aplicação como adsorvente e, por fim, promoverá também um impacto econômico em virtude da agregação de valor ao resíduo.

\section{REFERÊNCIAS}

CHAO H, ZHANG R, BAI F, LU PING, LIANG X, Removal of chromium (VI) from aqueous solutions using quaternized chitosan microspheres. Chinese J Chem Eng, v. 25, p. 153-158, 2017.

FREUNDLICH H.M.F, Uber die Adsorption in Losungen. Zeitschrift für Physikalische Chemie, v. 57, p. 385-470, 1906.

GAUTAM R K, MUDHOO A, LOFRANO G, CHATTOPADHYAYA M C, Biomassderived biosorbents for metal ions sequestration: Adsorbent modification and activation methods and adsorbent regeneration. J. Environ. Chem. Eng, v. 2, p. 239-259, 2014.

HO Y S, MCKAG Y (1998) A comparison of chemisorption kinetic models applied to pollutant removal on various sorbents. Process Saf. Environ. Protect, v. 76, p. 332-340, 1998.

LAGERGREN S, Zur theorie der sogenannten adsorption geloster stoffe. $K$. Sven. Vetenskapsakademiens. Handllingar, v. 24, p. 1-39, 1898.

LANGMUIR I, The adsorption of gases on plane surfaces of glasses and platinum. J.Am.Chem.Soc, v. 40, p. 1361-1403, 1918.

MOHAMMAD S, AL-MUSAWI, ESMAIL T G, HOSSEIN K, MANSUR Z, Adsorption performance of magnesium/aluminium layered double hydroxide nanoparticles for metronidazole from aqueous solution. Arab J Chem, v. 10, p. 611-623, 2018.

QI W, ZHAO Y, ZHENG X, JI M, ZHANG Z, Adsorption behavior and mechanism of Cr (VI) using Sakura waste from aqueous solution. Applied Surface Science, v. 360, p. 470-476, 2016.

QIU H, PAN L L, ZHANG Q J, ZHANG W, ZHANG Q, Critical review in adsorption kinetic models. J Zhejiang Univ-Sc A, v. 10, p. 716-724, 2009.

ZAREI S, MAHMOOD N, HOSSEIN R, The removal of Mercury ion pollution by using Fe3O4-nanocellulose: Synthesis, Characterization and DFT studies. J Hazard Mater, v. 344, p. 258-273, 2018. 